\title{
Influence of Nanocomposites of LDPE Doped with Nano-MgO by Different Preparing Methods on Its Dielectric Properties
}

\author{
Wenlong Zhang, ${ }^{1,2}$ Yajie Dai, ${ }^{1,2}$ Hong Zhao, ${ }^{1,3}$ and Lidong Zhong ${ }^{4}$ \\ ${ }^{1}$ Key Laboratory of Engineering Dielectric and Its Application, Ministry of Education, Harbin University of Science and Technology, \\ Harbin, Heilongjiang 150080, China \\ ${ }^{2}$ College of Material Science \& Engineering, Harbin University of Science and Technology, Harbin, Heilongjiang 150040, China \\ ${ }^{3}$ College of Electrical and Electronic Engineering, Harbin University of Science and Technology, Heilongjiang 150080, China \\ ${ }^{4}$ Zhongtian Technology Group Ltd., Nantong, Jiangsu 226000, China
}

Correspondence should be addressed to Hong Zhao; hongzhao@hrbust.edu.cn

Received 20 August 2014; Revised 2 November 2014; Accepted 18 November 2014

Academic Editor: Jian Sun

Copyright (c) 2015 Wenlong Zhang et al. This is an open access article distributed under the Creative Commons Attribution License, which permits unrestricted use, distribution, and reproduction in any medium, provided the original work is properly cited.

LDPE doped with nano-MgO can effectively suppress the space charge accumulation in the nanocomposite under DC applied field, and this suppression was affected greatly by the prepared nano- $\mathrm{MgO}$ particles. In this paper, the influence of nanocomposite of LDPE doped with nano-MgO by using different preparation methods on their space charge, and volume resistivity as well as DC breakdown strength was researched. The results showed that size of nano-MgO prepared by the traditional heating method was about $22.74 \mathrm{~nm}$, while being only about $12.76 \mathrm{~nm}$ by the microwave-assisted method. When the nano- $\mathrm{MgO}$ content in the composite was $2 \mathrm{wt} \%$ by weight, the space charge accumulation in this composite was obviously reduced; meanwhile both the volume resistivity and DC breakdown strength were enhanced. When nano-MgO was prepared by the microwave-assisted method, the electric properties of its LDPE nanocomposite were obviously better than those of nano-MgO prepared by traditional heating method.

\section{Introduction}

The contradiction between urban expansion and electricity demand is becoming more prominent. Long distance and effective and safe power transmission are the main developing direction to solve the conflict. Comparing direct current (DC) transmission with alternative current (AC) transmission, it has advantages of the lower line cost, lower power loss, transmission system stability, and so forth. But DC transmission of cross-linked polyethylene (XLPE) cable was hampered by its own shortcomings in which there is a lot of space charge accumulation leading to distorting electric field and even local electric breakdown of insulator of XLPE cable $[1,2]$. It was surprising that base polyethylene (base PE) doped with nano-MgO particles can effectively suppress the space charge accumulation. At the same time the composite still has excellent electrical properties. So researches of the dielectric polymer nanocomposite have become one of hot spots in electric power field [3].
Nanoparticles are generally agglomerated as a result of their high surface energy and therefore the disaggregation of nanoparticles in nanocomposites proves to be a key issue in obtaining good filler dispersion and to bring the nanoparticles into full play. However, there is different dispersion effect for the nanoparticles with different preparation methods. Nano-MgO particles prepared with direct precipitation method and homogeneous precipitation method as well as microwave-assisted method were researched in this paper. Nano-MgO particles prepared with direct precipitation method and homogeneous precipitation method have poor dispersion and serious aggregation. However, the microwave-assisted method has been used to prepare nanooxides, which has good dispersion and disaggregation, and so forth [4-6]. This paper systematically researched the fact that nano- $\mathrm{MgO}$ particle was prepared by microwaveassisted method and the influence of nano-MgO particles by different preparation methods on size, dispersion of nano$\mathrm{MgO}$, and dielectric properties of its nanocomposite. 


\section{Experimental Procedure}

2.1. Experimental Raw Materials. Magnesium nitrate hexahydrate $\left(\mathrm{MgNO}_{3} \cdot 6 \mathrm{H}_{2} \mathrm{O}\right)$ (Tianjin Guangfu Fine Chemical Research Institute, China), aqueous ammonia (37 wt\%) (Bazhou Chemical Plants, China), polyethylene glycol (PEG200) (Tianjin Regent Chemicals Co., Ltd.), urea $\left[\mathrm{CO}\left(\mathrm{NH}_{2}\right)_{2}\right]$ (Tianjin Chemical Reagent Co., Ltd., China), ammonium bicarbonate (Bazhou City Chemical Plant, Hubei China), LDPE (BW150, BASF YPC Co., Ltd., China), and EVA (EF-443, 14\%, LG Chemical Company, South Korea) are used in this research.

\subsection{Preparation of $\mathrm{Mg}(\mathrm{OH})_{2}$}

2.2.1. Direct Precipitation Method. $\mathrm{MgNO}_{3} \cdot 6 \mathrm{H}_{2} \mathrm{O}$ was dissolved in distilled water $(200 \mathrm{~mL})$ to prepare a solution of certain concentration $(0.2 \mathrm{~mol} / \mathrm{L})$ in a three-necked bottle. Then appropriate amount of aqueous ammonia was put into the three-necked bottle drop by drop at starting heating by a sheathed heater. White precipitate powder began to occur after dropwise addition of aqueous ammonia in the solution of the flask. After maintaining a certain period of time for heating the solution, the white precipitate powder was washed with water and alcohol and then $\mathrm{Mg}(\mathrm{OH})_{2}$ powder was obtained. See $[7,8]$.

2.2.2. Homogeneous Precipitation Method. $\mathrm{MgNO}_{3} \cdot 6 \mathrm{H}_{2} \mathrm{O}$ was dissolved in distilled water $(200 \mathrm{~mL})$ to prepare a solution of certain concentration $(0.2 \mathrm{~mol} / \mathrm{L})$ in a three-necked bottle. Then, magnesium nitrate aqueous solution was put into a three-necked flask. Once heated, a small amount of PEG dispersant $(0.1 \mathrm{~mL})$ was added into the flask and then stirred for $5 \mathrm{~min}$; finally, urea $(1.0 \mathrm{~g})$ was added according to the predetermined ratio. After $6 \mathrm{~h}$ reaction at $125^{\circ} \mathrm{C}$, a white precipitate was formed which was washed by water and alcohol, respectively, with filtration devices. Then the precipitate was put into a vacuum dry oven (at $80^{\circ} \mathrm{C}, 24 \mathrm{~h}$ ) to dry to obtain $\mathrm{Mg}(\mathrm{OH})_{2}$ powder. See [9].

2.2.3. Microwave-Assisted Method. A certain amount of ammonium bicarbonate accompanied with a small amount of PEG200 (1 mL) was dissolved in distilled water $(20 \mathrm{~mL})$. Then the three-necked flask containing the solution was heated by microwave oven under $80^{\circ} \mathrm{C}, 625 \mathrm{~W}$ for $30 \mathrm{~min}$. Then, magnesium nitrate solution was added into the flask step by step with a separatory funnel at a fixed speed of dropping in accordance with the addition of molar ratio (1:4) of magnesium nitrate and ammonium bicarbonate. A white precipitate was produced at $50 \mathrm{~min}$ and $80^{\circ} \mathrm{C}$. And washed it with water and alcohol, respectively, with suction device. Finally, the precipitate was put into a vacuum dry oven (at $80^{\circ} \mathrm{C}, 24 \mathrm{~h}$ ) to dry to obtain $\mathrm{Mg}(\mathrm{OH})_{2}$ powder.

2.3. Preparation of Surface Coupling Treatment of Nano-MgO. Nano-MgO was prepared by stepwise calcination. First, $\mathrm{Mg}(\mathrm{OH})_{2}$ was put into the muffle furnace and calcinated at the temperature of $300-400^{\circ} \mathrm{C}$ for 1 hour. Then, it was taken out for ball milling in planetary ball mill at rotating speed of
$200 \mathrm{rpm}$ for $20 \mathrm{~min}$ (forward $10 \mathrm{~min}$, reverse $10 \mathrm{~min}$, resp.). Secondly, it was put into muffle furnace and calcinated again at the temperature of $500^{\circ} \mathrm{C}$ for 2 hours. Then it was fetched out and the nano-MgO powder was obtained. Nano-MgO particles' surface was treated with silane coupling agent $[\mathrm{KH}$ 550, $\mathrm{NH}_{2} \mathrm{CH}_{2} \mathrm{CH}_{2} \mathrm{CH}_{2} \mathrm{Si}\left(\mathrm{OC}_{2} \mathrm{H}_{5}\right)_{3}, 2$ wt $\%$ of the particle loading] at about $60^{\circ} \mathrm{C}$ for $16 \mathrm{~min}$ (forward $8 \mathrm{~min}$, reverse $8 \mathrm{~min}$, resp.) using ball milling at a ball speed of $140 \mathrm{rpm}$.

2.4. Preparation of LDPE Nanocomposite. LDPE (40 g) and nano-MgO particle treated by using silane coupling agent ( $2 \mathrm{wt} \%$, nano-MgO prepared by microwave-assisted method or direct precipitation method or homogeneous precipitation method, resp.) were blended to prepare three kinds of nanocomposites at $150^{\circ} \mathrm{C}$ and $80 \mathrm{rpm}$ for $15 \mathrm{~min}$ in the mixer of torque rheometer. During the blending process, ethylenevinyl-acetate copolymer (EVA, $9 \mathrm{wt} \%$ of LDPE loading) was added into the mixer in order to further improve the dispersibility between nano-MgO and LDPE in the nanocomposite.

2.5. Analysis by SEM. As for sample of the composite, it must be broken into small pieces, whereas the powder of $\mathrm{MgO}$ can be used directly. The brittle fracture processing of the composite sample was carried out in liquid nitrogen. After that, the fragments of nanocomposite sample or the nano$\mathrm{MgO}$ powders were fixed on the tin block with conductive adhesive and then coated with gold. Finally, the dispersion and size of nano-MgO particles were observed by using a scanning electron microscope (SEM, FEI Sirion 200, Dutch Philips Co., Ltd.).

2.6. XRD Test Method. The size of nano-MgO powder was analyzed by X-ray diffractometer (XRD, model D/max 2500, Rigaku Co., Ltd., Japan), whose scanning speed was $4^{\circ} / \mathrm{min}$ and angle range was $10^{\circ}-90^{\circ}$. The size of nano-MgO powder was calculated according to the Scherrer formula:

$$
D=\frac{K \lambda}{(L \cos \theta)} \text {. }
$$

In the formula, $K=0.89$ and $\lambda=0.15406 \mathrm{~nm}$. $L$ stands for width at half-intensity diffraction line degree; $\theta$ for a diffraction angle $\left({ }^{\circ}\right) ; D$ for grain size $(\mathrm{nm})$.

2.7. Volume Resistivity Test. Volume resistance test of the composite specimen slice which was about $1 \mathrm{~mm}$ in thickness was carried by digital resistance meter (ZC-36) under testing voltage being $1000 \mathrm{~V}$ applied to specimen slice at threeelectrode system (Figure 1), and the volume resistivity was calculated according to formula (2), in which $\rho_{v}(\Omega \cdot \mathrm{m})$ was the resistivity, $R_{v}(\Omega)$ the composite volume resistance, $d_{1}$ $(\mathrm{m})$ the protested electrode diameter, $g(\mathrm{~m})$ the electrode gap between the protested electrode and the shied electrode, and $h(\mathrm{~m})$ the specimen thickness:

$$
\rho_{v}=R_{v} \frac{\pi\left(d_{1}+g\right)^{2}}{4 h} .
$$


TABLE 1: The volume resistivity of the composites.

\begin{tabular}{lcccc}
\hline Item & Base LDPE & $\begin{array}{c}\text { LDPE doped with MgO } \\
\text { prepared by microwave } \\
\text { method }\end{array}$ & $\begin{array}{c}\text { LDPE doped with MgO } \\
\text { prepared by direct } \\
\text { precipitation method }\end{array}$ & $\begin{array}{c}\text { LDPE doped with MgO } \\
\text { prepared by homogeneous } \\
\text { precipitation method }\end{array}$ \\
\hline Volume resistivity $/ \Omega \cdot \mathrm{m}$ & $8.49 \times 10^{12}$ & $1.23 \times 10^{15}$ & $1.27 \times 10^{14}$ & $2.33 \times 10^{14}$ \\
\hline
\end{tabular}

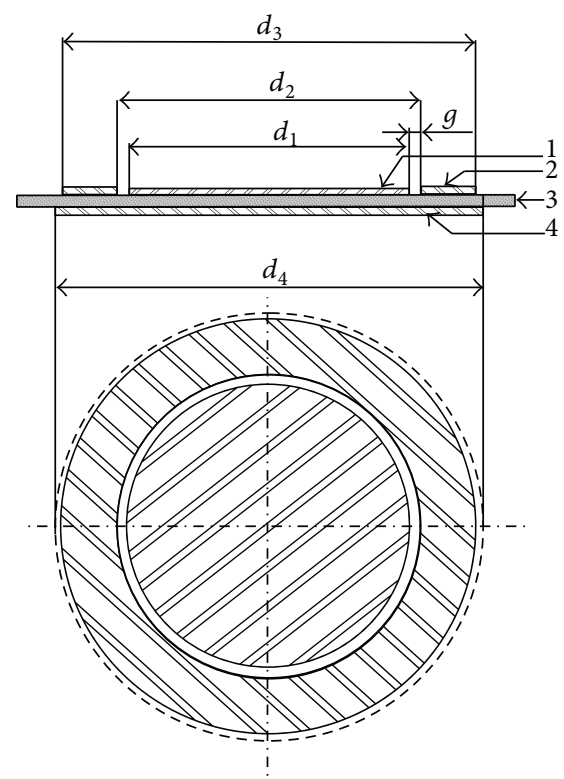

Figure 1: Three-electrode system. (1) Protected electrode, (2) shield electrode, (3) specimen slice, and (4) no protecting electrode; $\left(d_{1}\right)$ protected electrode diameter $(50 \mathrm{~mm}),\left(d_{2}\right)$ shield electrode inner diameter $(54 \mathrm{~mm}),\left(d_{3}\right)$ shield electrode external diameter $(74 \mathrm{~mm})$, and $\left(d_{4}\right)$ no protecting electrode diameter $(\geq 74 \mathrm{~mm})$. $(g)$ electrode gap between the protected electrode and shield electrode $(2 \mathrm{~mm})$.

2.8. DC Breakdown Strength Test. The composite circular specimen slice (the sample thickness of $100 \mu \mathrm{m}$, the diameter of about $10 \mathrm{~cm}$ ) was put into transformer oil. Breakdown field value was detected by the breakdown voltage tester (HT-100) and at continuous elevating speed voltage of $3 \mathrm{kV} / \mathrm{s}$.

2.9. Space Charge Test. Space charge in the sample was tested by pulsed electro-acoustic-01 (PEA-01) under the strength of electric field being $40 \mathrm{kV} / \mathrm{mm}$.

\section{The Results and Discussion}

3.1. SEM Analysis of Nano-MgO. Nano- $\mathrm{MgO}$ was made from $\mathrm{Mg}(\mathrm{OH})_{2}$ by two-step calcination method in muffle furnace, whereas the $\mathrm{Mg}(\mathrm{OH})_{2}$ was made by microwave-assisted method, direct precipitation method, and homogenous precipitation method, respectively. The dispersion and size of the nano-MgO particles made by three methods were observed by using scanning electron microscope (SEM), as shown in Figures 2(a), 2(b), and 2(c). The results indicated that the diameter size of $\mathrm{MgO}$ particle prepared by microwaveassisted method was about $49 \mathrm{~nm}$, which was the minimum of the nano- $\mathrm{MgO}$ particles and disperses uniformly among three methods. In addition, the particles agglomeration phenomenon was not obvious (see Figure 2(a)), while the diameter size of $\mathrm{MgO}$ particle prepared by direct precipitation method was beyond the nanometer size, and it had serious agglomeration phenomenon (see Figure 2(b)).

The diameter size of $\mathrm{MgO}$ powder prepared by homogenous precipitation method ranges from 70 to $90 \mathrm{~nm}$ and it had obvious agglomeration (see Figure 2(c)). The reasons may be that the uniformity of particle size is influenced by multiple nucleation of precipitate phase. Different from the direct precipitation method and homogeneous precipitation method, the microwave-assisted method can provide uniform heat in a very short period of time to greatly eliminate the temperature gradient and instantaneously nucleate for precipitate phase of $\mathrm{Mg}(\mathrm{OH})_{2}$ which was calcined by two-step method in muffle furnace to obtain uniform distribution and dispersion of nano-MgO particles.

3.2. XRD Analysis of Nano-MgO. The nano-MgO powder prepared by microwave-assisted was compared with conventional heating methods by using X-ray diffraction (XRD) in Figure 3. The $2 \theta$ values of the diffraction peaks of nano$\mathrm{MgO}$ particles prepared by microwave-assisted method and conventional method were basically the same and the crystals were both cubic crystal structure from Figure 3. According to Scherer formula (1) calculation, the size of nano-MgO prepared by microwave-assisted method was about $12.76 \mathrm{~nm}$, and the traditional one was $22.74 \mathrm{~nm}$.

3.3. Influence of Nano-MgO Preparation Method on the Volume Resistivity of Composites. The nano-MgO loading was fixed at $2 \mathrm{wt} \%$, and nano-MgO prepared by different methods was doped into LDPE. The impact on the volume resistivity of composites was investigated and the results were shown in Table 1 .

As can be seen from Table 1, after LDPE doped with $\mathrm{MgO}$ nano-MgO was prepared by microwave-assisted method, the volume resistivity of composites was the maximum. The reason may be attribuited to the good dispersion of nano$\mathrm{MgO}$ in LDPE matrix. Nano-MgO can limit the movement of molecular chain effectively, forming a lot of traps, and enhance the ability to capture charge under applying the electric field. Therefore, the volume resistivity of composites was higher than other nano-MgO composites $[10,11]$.

3.4. Effect of Nano-MgO by Prepared Methods on the DC Breakdown Strength of Composite. The nano-MgO loading was fixed as $2 \mathrm{wt} \%$; using nano-MgO by different preparation methods in LDPE matrix, the impact on DC breakdown 
TABLE 2: DC breakdown strength of the composites.

\begin{tabular}{lcccc}
\hline Item & Base LDPE & $\begin{array}{c}\text { LDPE doped with MgO } \\
\text { prepared by microwave } \\
\text { method }\end{array}$ & $\begin{array}{c}\text { LDPE doped with MgO } \\
\text { prepared by direct } \\
\text { precipitation method }\end{array}$ & $\begin{array}{c}\text { LDPE doped with MgO } \\
\text { prepared by homogeneous } \\
\text { precipitation method }\end{array}$ \\
\hline Breakdown strength $/ \mathrm{kV} / \mathrm{mm}$ & 260.9 & 310.0 & 263.3 & 270.4 \\
\hline
\end{tabular}

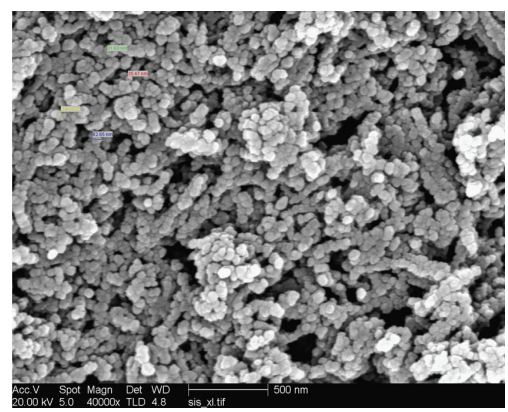

(a)

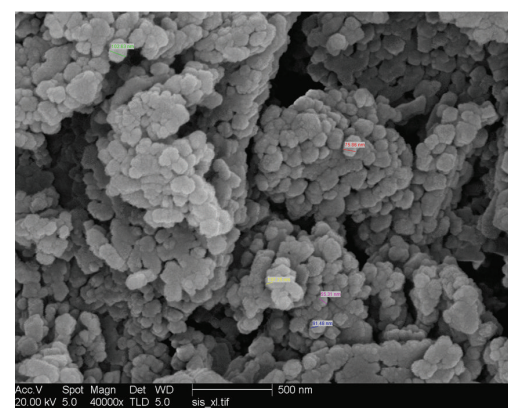

(b)

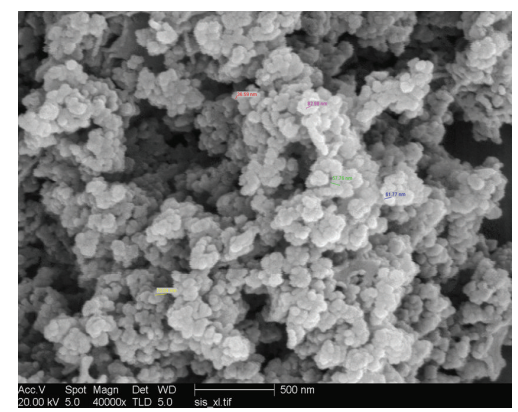

(c)

FIGURE 2: (a) Nano-MgO by microwave-assisted method; (b) nano-MgO by direct precipitation method; (c) nano-MgO by homogenous precipitation method.

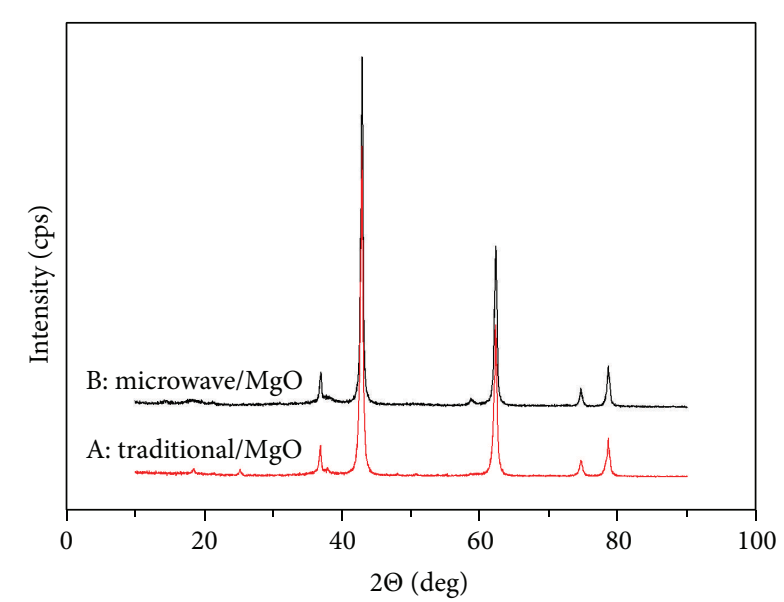

FIGURE 3: XRD pattern of nano-MgO prepared by microwaveassisted and conventional methods, respectively.

strength of the composite was investigated. The results were shown in Table 2.

As can be seen from Table 2, DC breakdown strength of the composites has increased in comparison with LDPE without adding nano-MgO. However, the LDPE blended with $\mathrm{MgO}$ prepared by microwave-assisted method has the maximum DC breakdown field strength due to its perfect dispersion. There was the Coulomb blockade effect, which will limit the directional movement of carriers. On the other hand, it will result in LDPE matrix stretched to a certain extent. Stretching causes the lattice of LDPE dislocations which produce more and deeper traps. At the same time, the ability to trap electrons will be enhanced [12].

3.5. Influences of Nano-MgO Preparation Methods on Space Charge in the Composites. Nano-MgO was fixed at $2 \mathrm{wt} \%$ by weight. Using of nano-MgO blended with LDPE to form the composite, the effect of the space charge distribution on the composite was investigated. The experimental results were shown in Figure 4.

From Figure 4(a), it can be seen that base LDPE has a large number of homocharges injected near the cathode and anode. Comparing Figure 4(b) with Figures 4(c) and 4(d), composite doped with nano-MgO prepared by microwaveassisted method has the anode and cathode without the homocharges injected. It almost has no space charge in the sample with effective effect to suppress space charge in composite. This may be attributed to the fact that the diameter of nano-MgO prepared by microwave-assisted method was small and well distributed in the composite due to good compatibility with LDPE matrix. It will enhance the charge injection barrier and at the same time reduce the mobility of the charge because of the particle surface charge layer effects. So the effect of space charge suppression was the best $[2,3]$.

\section{Conclusions}

In this paper, nano-MgO prepared by microwave-assisted method was compared with ones prepared by conventional methods. The former has smallest particle size and no obvious agglomeration. Electric properties of LDPE doped with the nano-MgO prepared by microwave-assisted method have been greatly improved by contrast with LDPE doped with the nano-MgO prepared by conventional method.

\section{Conflict of Interests}

The authors declare that there is no conflict of interests regarding the publication of this paper. 


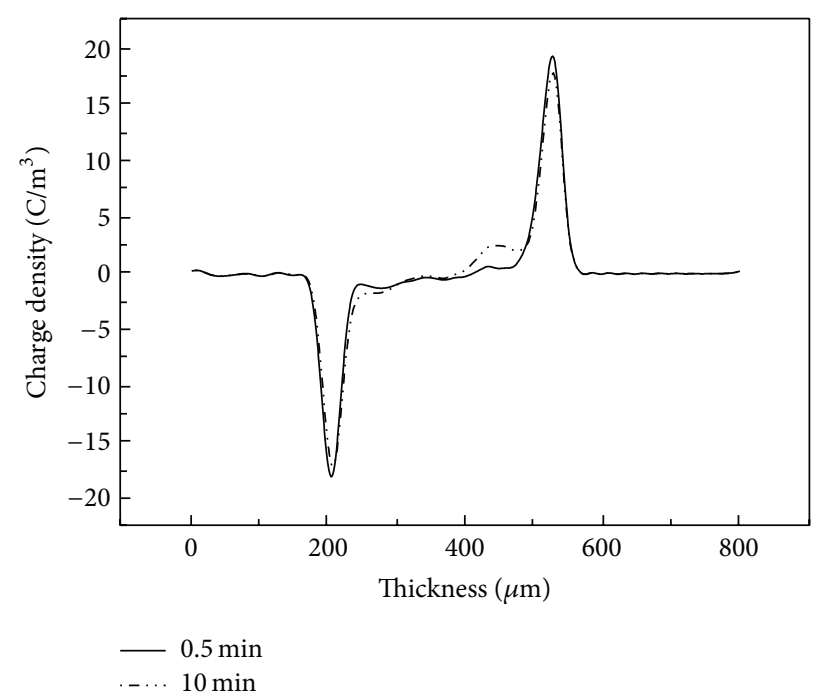

(a) Charge distribution of base LDPE

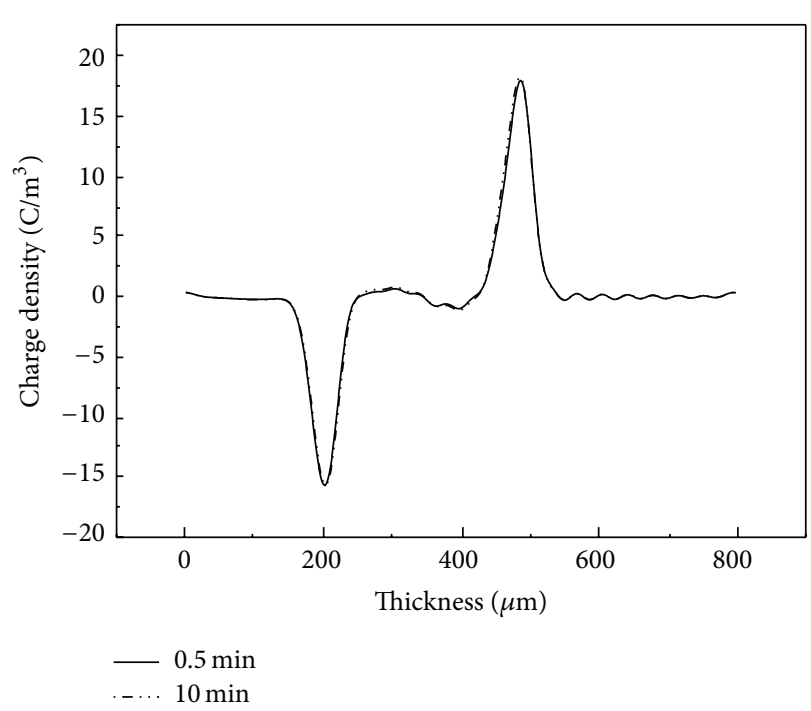

(c) Charge distribution of LDPE doped with MgO prepared by direct precipitation method

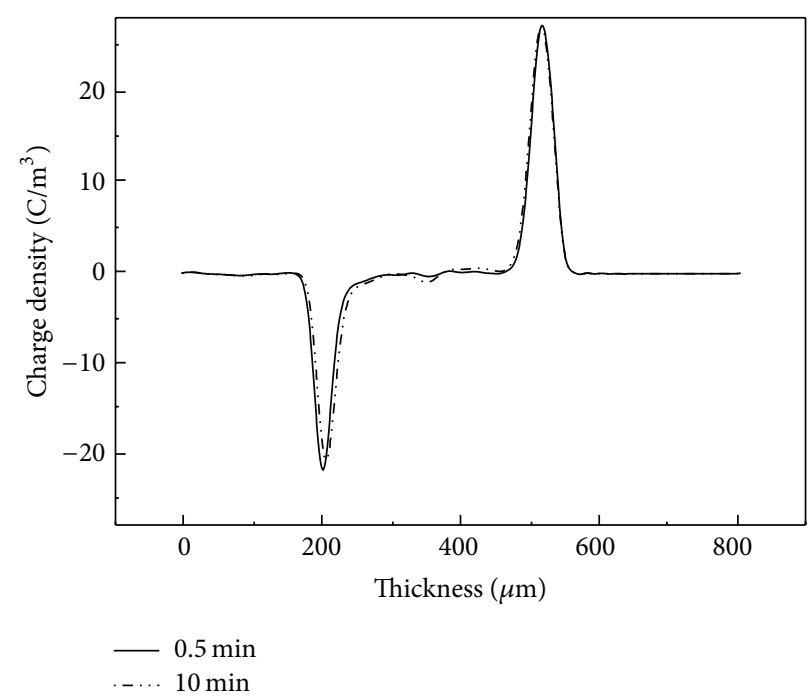

(b) Charge distribution of LDPE doped with $\mathrm{MgO}$ prepared by microwave-assisted method

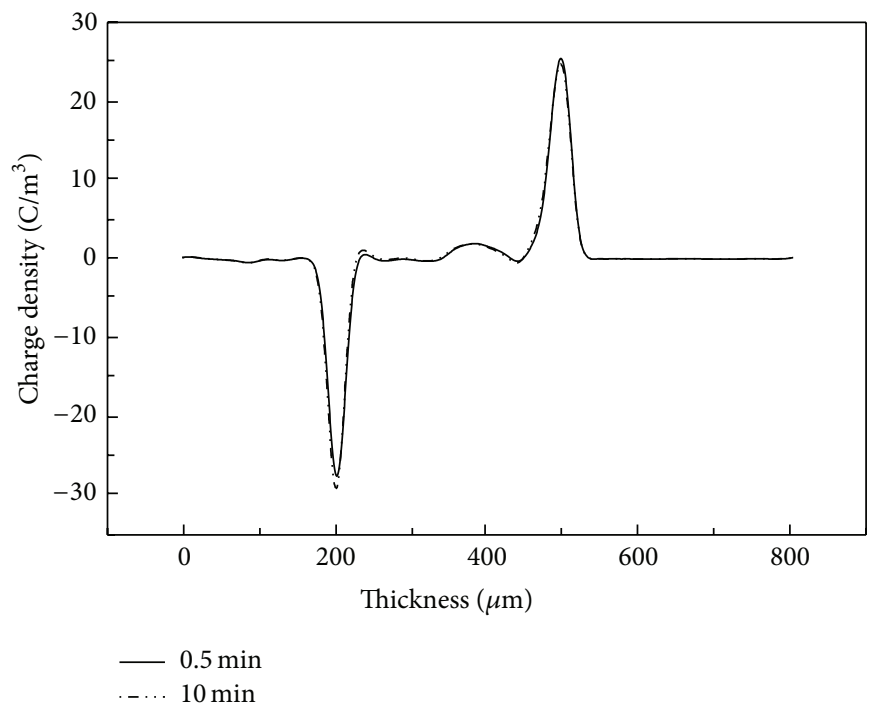

(d) Charge distribution of LDPE doped with MgO prepared by homogeneous precipitation method

FIgURE 4: Charge distribution of base LDPE and its nano-MgO composite.

\section{Acknowledgment}

The authors thank National Science Foundation of China (Grant no. 51337002) for their financial support.

\section{References}

[1] I. L. Hosier, A. S. Vaughan, and S. G. Swingler, "An investigation of the potential of ethylene vinyl acetate/polyethylene blends for use in recyclable high voltage cable insulation systems," Journal of Materials Science, vol. 45, no. 10, pp. 2747-2759, 2010.

[2] G. C. Montanari and P. H. F. Morshuis, "Space charge phenomenology in polymeric insulating materials," IEEE Transactions on Dielectrics and Electrical Insulation, vol. 12, no. 4, pp. 754-767, 2005.
[3] K. Wu, X. Chen, X. Wang et al., "Research of polyethylene nanocomposites for HVDC cables," Insulation Materials, vol. 43, no. 4, pp. 1-2, 2010.

[4] D.-z. Wang, Y.-j. Sun, X.-y. Liu et al., "The process research on preparation of nano-Magnesia," Powder Metallurgy Industry, vol. 22, no. 6, pp. 49-52, 2012.

[5] Y. Li, P. Wang, and H. L. Cao, "Microwave assisted synthesis of n-butyl lactate catalyzed by acid-activatedmont morillonite clay," Material Science and Technology, vol. 20, no. 1, pp. 49-57, 2012.

[6] H. Wang, P. Wang, and J.-F. Zhang, "Photocatalysis activity and preparation of Fe-doped $\mathrm{TiO}_{2}$ hydrosol by microwave irradiation peptization," Material Science and Technology, vol. 18, no. 4, pp. 545-554, 2010. 
[7] Z.-Q. Liu, L.-J. Li, Z.-M. Zeng, F. Nie, L.-Y. Chai, and Y.-F. Chen, "Preparation of monodispersed and hexagonal magnesium hydroxide from bischofite," Materials Science and Technology, vol. 19, no. 8, pp. 138-143, 2011.

[8] K. Xu, B.-L. Zhang, C.-H. Hou et al., "Review on nano-magnesia preparation technology," Inorganic Chemicals Industry, vol. 39, no. 6, pp. 7-9, 2007.

[9] W.-L. Zhang, J.-W. Xu, C. Ji et al., "Preparation of nanomagnesia by homogeneous precipitation," Journal of Harbin University of Science and Technology, vol. 15, no. 6, pp. 112-115, 2010.

[10] S.-Q. Chen, X. Chen, X. Wang et al., "tudy of space charge distribution and volume resistivity in composite of polyethylene/nano zinc oxide," Insulation Materials, vol. 40, no. 2, pp. 48-53, 2007.

[11] Y. Murakami, M. Nemoto, S. Okuzumi et al., "DC conduction and electrical breakdown of MgO/LDPE nanocomposite," IEEE Transactions on Dielectrics and Electrical Insulation, vol. 15, no. 1, pp. 33-38, 2008.

[12] Y. Wang, M. Xu, Y. Sun et al., "Study on the resistance and electrical breakdown properties of nano silver/epoxy resin composite," Insulation Materials, vol. 39, no. 4, pp. 37-40, 2006. 

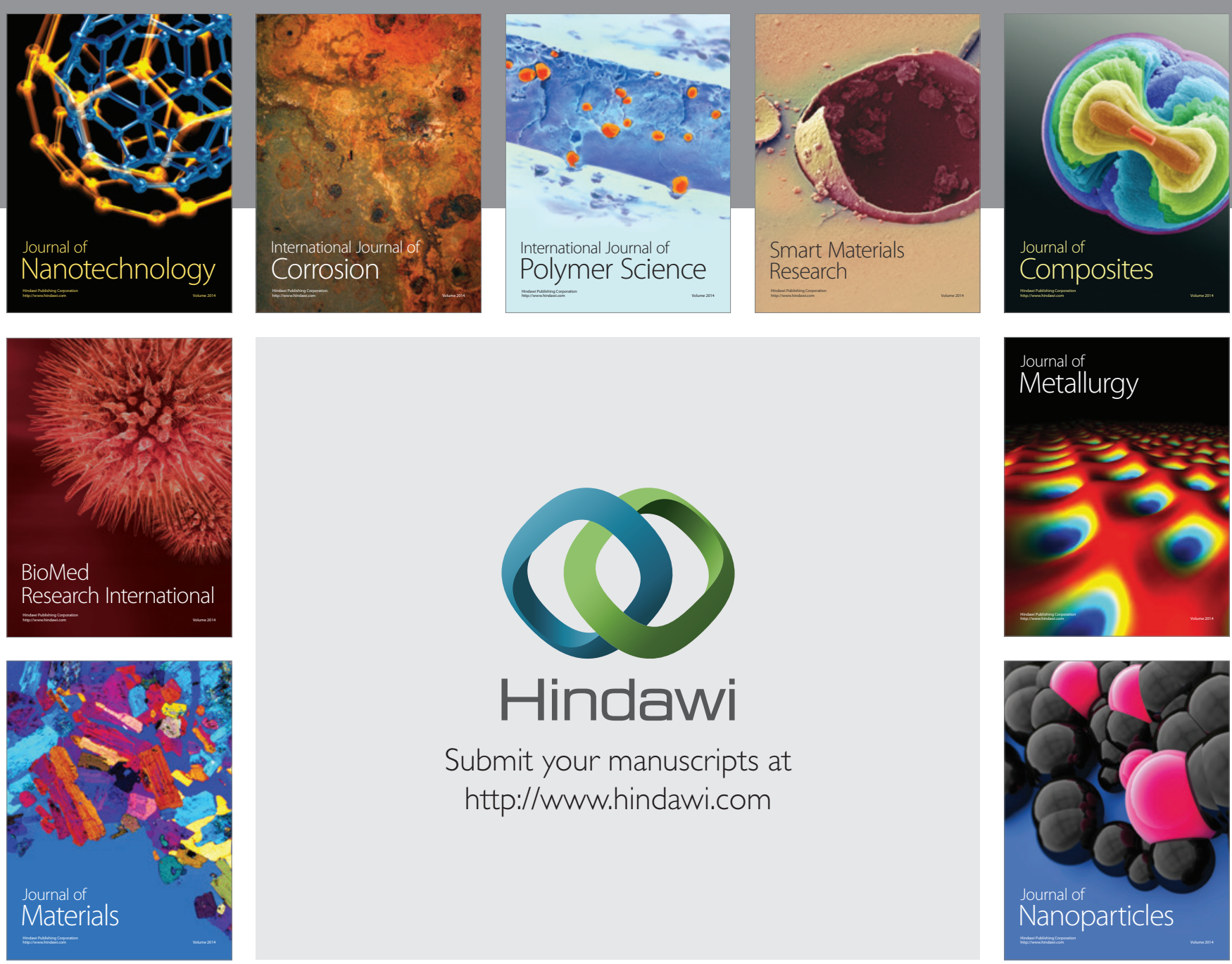

Submit your manuscripts at http://www.hindawi.com
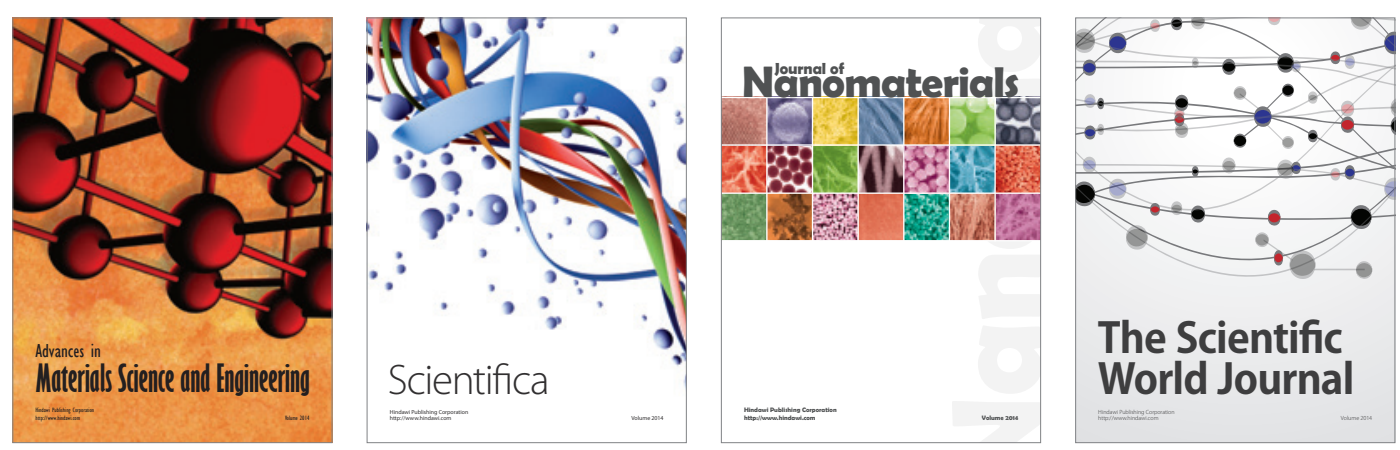

\section{The Scientific World Journal}
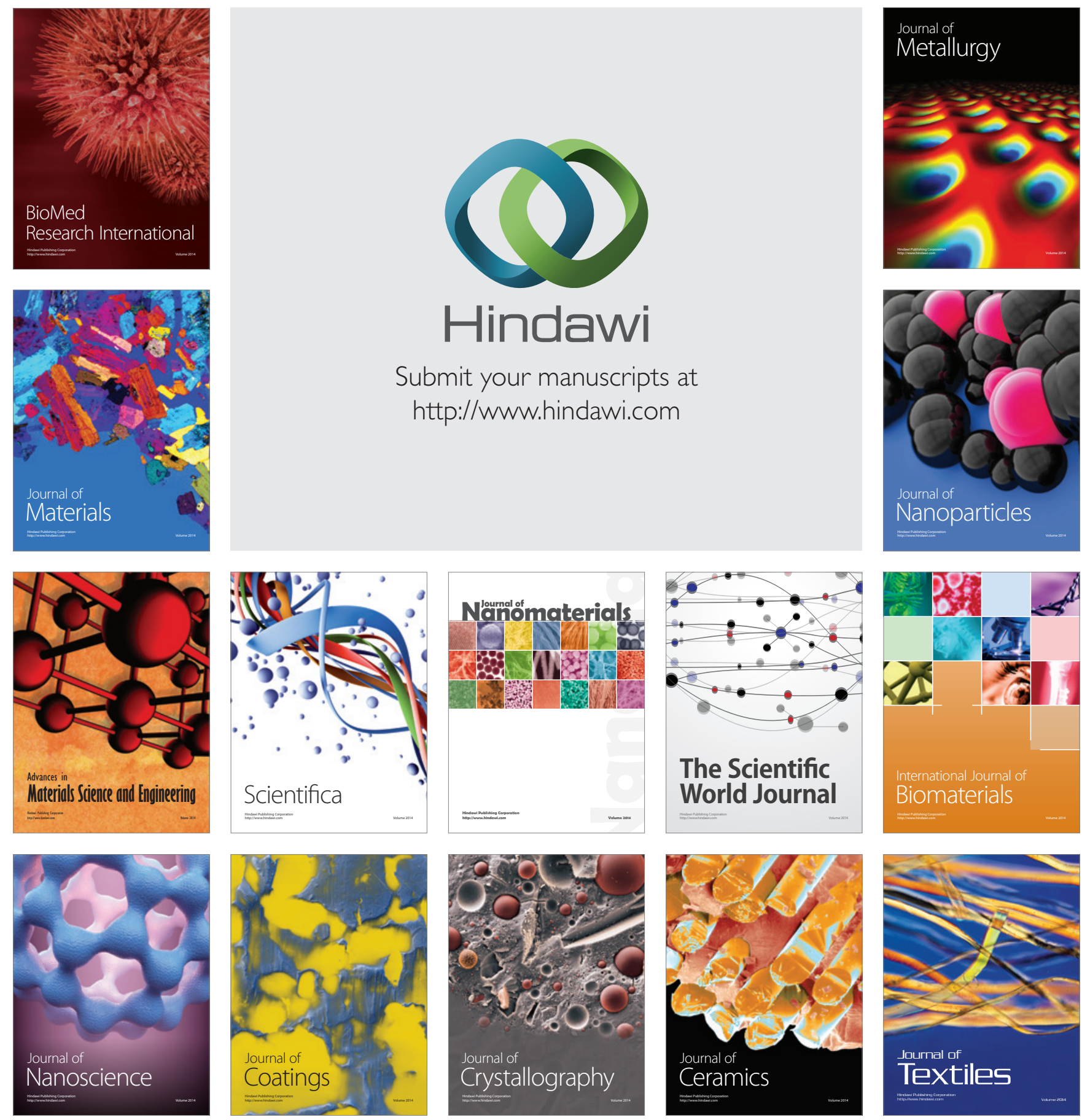